\title{
The Calculations of Evolving Rates Realized with Two of Type Variables in Whole Process for Elastic-Plastic Materials Behaviors under Unsymmetrical Cycle
}

\author{
Yangui $\mathrm{Yu}^{1}$ \\ ${ }^{1}$ Zhejiang Guangxin New Technology Application Academy of Electromechanical and Chemical Engineering, \\ Hangzhou, China \\ Correspondence: Yangui Yu, Zhejiang Guangxin New Technology Application Academy of Electromechanical \\ and Chemical Engineering, 13 Eeast Edifice, 160 International Garden Tiannushan Road, Hangzhou 310007, \\ China. E-mail: ygyu@vip.sina.com,gx_yyg@126.com
}

Received: June 30, 2012 Accepted: July 23, 2012 Online Published: September 6, 2012

doi:10.5539/mer.v2n2p77 URL: http://dx.doi.org/10.5539/mer.v2n2p77

\begin{abstract}
On the basis of fatigue, damage and fracture accidents happened from engineering practices, based on data of references to research and analyze, research some mathematical methods in mechanics, adopt two types of variables $a$ and $D$, combine program computing of computer, for elastic-plastic steels with short and long cracks, for their fatigue-damage-fracture behaviors put forward several calculations models, which are the crack growth rates and the damage evolving rates at each stage and in whole process; Also provide calculations methods about the two types of variables, some methods calculated in two stages or in whole process, and the conversion methods between the variables, between the equations and between the dimensional units; Indicate their physical and the geometrical meanings for some key parameters; Also give a calculation example about short crack and long crack growth rates and damage evolving rates in two stages. Thereby communicate each other the intersecting relations between their computing models and between the disciplines. This works for saving man powers and funds on fatigue-damage-fracture testing, promote applying of each discipline, it will be having practical significance.
\end{abstract}

Keywords: two types of variables, driving force, elastic-plastic materials, physical and geometrical meaning, comprehensive material constants, rate in whole process

\section{Introduction}

Adopt the crack size $a$ as a variable in the fracture mechanics to describe crack growth process, and adopt the damage variable $D$ in the damage mechanics to describe a damage evolving process. If can convert each other for their relations between the damage variables, between the equations and between the dimensional units, which are describe the material behaviors in varied disciplines, and if can provide respectively some conversion methods, thus we are also able to adopt the same variables $a_{1}$ and $a_{2}$ or the same variables $D_{1}$ and $D_{2}$ to calculate the crack growth rates or damage evolving rates by their curves at each stage. Even we can also adopt variable $a$, or variable $D$ to describe their behaviors in overall process. That way, it will be having practical significance for stint man powers and funds in fatigue-damage-fracture testing, for promoting developing and applying of each discipline.

\section{The Calculations of the Evolving Rates of Each Stage for Elastic-plastic Materials with Cracks}

For some elastic-plastic materials with short cracks $a_{l}$, calculations for short cracks growth processes, or calculations for their damage evolving processes can be imitated by means of method in reference (Smith $\&$ Watson, 1970), that is to realize damage calculations by multiplication parameters $\Delta \varepsilon_{n} \Delta \sigma$ with plastic strain range $\Delta \varepsilon_{n}$ and stress range $\Delta \sigma$ at the crack forming stage (the first stage). For long crack $a_{2}$, this paper also adopt the multiplication parameters $\Delta \delta_{t} \Delta K_{l}$ with the crack tip open displacement range $\Delta \delta_{t}$ and the stress intensity factor range $\Delta K_{l}$ at the crack growth stage (the second stage), thereby achieve calculations of damage evolving rates or crack growth rates at each stage and even in the whole process. 


\subsection{The Calculations of Rates at First Stage under Mean Stress $\sigma_{m} \neq 0$ Condition}

The curve of growth rate for a short crack $a_{1}$ at the first stage is just described with curve $5\left(\sigma_{m} \neq 0\right)$ in the positive direction coordinate system in Figure 1 (Yu \& Sun, 2011; Yu \& Bi, 2009). And equation of its rate is as following form Yu and Jiang (2002).

$$
d a_{1} / d N_{1}=A_{1}^{*} \cdot Q_{1}^{\frac{m_{1} m_{1}^{\prime}}{m_{1}+m_{1}^{\prime}}} \quad(\mathrm{m} / \text { cycle })
$$

Where

$$
\begin{gathered}
Q_{1}=(\varepsilon \cdot \sigma) \cdot a_{1}^{1 /\left(\frac{m_{1} m_{1}^{\prime}}{\left.m_{1}+m_{1}^{\prime}\right)}\right)},\left(\% M P a \cdot \sqrt{\frac{m_{1} m_{1}^{\prime}}{m_{1}+m_{1}^{\prime}}} \sqrt{m}\right), \\
\Delta Q_{1}=(\Delta \varepsilon \cdot \Delta \sigma) \cdot a_{1}^{1 /\left(\frac{m_{1} m_{1}^{\prime}}{\left.m_{1}+m_{1}^{\prime}\right)},\right.}
\end{gathered}
$$

The parameters $Q_{l}$ and $\Delta Q_{l}$ are defined severally as the short-crack stress-strain factor and the stress-strain factor range, that are driving force of short crack growth. Their units are $M P a \cdot \sqrt[\frac{m_{l} m_{1}^{\prime}}{m_{1}+m_{1}^{\prime}}]{m}$. The parameters $m_{l}$ and $m_{l}{ }^{\prime}$ are respectively material constants under high cycle or low cycle fatigue. The $\mathrm{m}_{1}=-1 / b_{1}{ }^{\prime}, b_{1}{ }^{\prime}$ is the fatigue strength exponent under high cycle fatigue; the $m_{l}{ }^{\prime}=-1 / c_{l}{ }^{\prime}, c_{l}{ }^{\prime}$ is the fatigue ductility exponent under low cycle fatigue.

$$
A_{1}^{*}=2\left[4\left(\sigma_{f}^{\prime}-\sigma_{m}\right)\left(\varepsilon_{f}^{\prime}{ }_{f}-\varepsilon_{m}\right)\right]^{-\frac{m_{1} m_{1}^{\prime}}{m_{1}+m_{1}^{\prime}}} \frac{\ln [1 /(1-\varphi)]}{\left(N_{1 f}-N_{01}\right)},\left(\mathrm{MPa}^{-\frac{m_{1} m_{1}^{\prime}}{m_{1}+m_{1}^{\prime}}} / \text { cycle }\right)
$$

Where, the parameter $A_{l}{ }^{*}$ is a comprehensive material constant. Under mean stress $\sigma_{m} \neq 0$ condition, it can be corrected by the Morrow's method (Morrow, 1968), but must be obtained from experiment. Author research and think, physical meaning of the $A_{l}{ }^{*}$ is a concept of power, it is an energy gave out that is a material to resist external force, just is a maximal increment value gave out energy to cause failure in one cycle; Its geometrical meaning is a maximal micro-trapezium area to approximate to beeline, that is a projection of corresponding to curve 5 on the y-axis (Figure 1), also is an intercept between $O_{1}-O_{3}$. Its slope of micro-trapezium bevel edge just is corresponding to the exponent $\frac{m_{1} m_{1}}{m_{1}+m_{1}}$ of the formula (4). And the comprehensive material constant $A_{l}{ }^{*}$ is having a function relation with other parameters in (4) (Yu \& Jiang, 2002). $\sigma_{f}^{\prime}$ is a fatigue strength coefficient, $\varepsilon_{f}^{\prime}$ is a fatigue ductility coefficient. $\varepsilon_{m}$ is mean strain, it can ignore, $\varepsilon_{m} \approx 0 . \varphi$ is a reduction of area in material mechanics. $N_{0 I}$ is initial life at first stage, $N_{0 I}=0 . N_{l f}$ is failure life, $N_{l f}=1$ It should point that fraction $\ln [1 /(1-\phi)] /\left(N_{1 f}-N_{01}\right)$ in (4) to equal $v_{f}$ as below

$$
v_{f}=\ln [1 /(1-\varphi)] /\left(N_{1 f}-N_{01}\right)=\ln [1 /(1-\varphi)],(\% / \text { cycle })
$$

It should point that its physical meaning of the (5) is a rate of whole failure to cause specimen material in a cycle, its unit is this \%/cycle. So final expansion equation for (1) is as follow form (Yu \& Xu, 2007; Yu \& Liu, 2006; Yu \& Li, 2007; Yu, 2003; Yu \& Zhao, 1999).

$$
d a_{1} / d N_{1}=2\left[4\left(\sigma_{f}^{\prime}-\sigma_{m}\right)\left(\varepsilon_{f}^{\prime}-\varepsilon_{m}\right)\right]^{-\frac{m_{1} m_{1}^{\prime}}{m_{1}+m_{1}^{\prime}}} \times v_{f} \times\left(\Delta \varepsilon_{p} \Delta \sigma\right)^{\frac{m_{1} m_{1}^{\prime}}{m_{1}+m_{1}^{\prime}}} \times a_{1},
$$

On the other hand, if use the damage variable $D_{l}$ to express, that is equivalent with above mentioned Equations (1) and (6), then equations of damage evolving rates should be below as modes (7) and (8).

$$
\begin{gathered}
d D_{1} / d N_{1}=A_{1}^{*} \cdot Q_{1}{ }^{\frac{m_{1} m_{1}^{\prime}}{m_{1}+m_{1}^{\prime}}},(\mathrm{D} / \text { cycle }) \\
d D_{1} / d N_{1}=2\left[4\left(\sigma_{f}^{\prime}-\sigma_{m}\right)\left(\varepsilon_{f}^{\prime}-\varepsilon_{m}\right)\right]^{-\frac{m_{1} m_{1}^{\prime}}{m_{1}+m_{1}^{\prime}}} \times v_{f} \times\left(\Delta \varepsilon_{p} \Delta \sigma\right)^{\frac{m_{1} m_{1}^{\prime}}{m_{1}+m_{1}^{\prime}}} \times D_{1},
\end{gathered}
$$

Where the parameter $Q_{l}$ in (1) should convert corresponding to $Q_{l}^{\prime}$, the parameters $Q_{l}^{\prime}$ ' and $\Delta Q_{l}$ ' are defined severally as the damage stress-strain factor and the damage stress-strain factor range, that are also driving forces of damage evolving of materials at first stage. Its unit of the $Q_{l}{ }^{\prime}$ is this $M P a \cdot D^{1 / \frac{m_{1} m_{1}^{\prime}}{m_{1}+m_{1}^{\prime}}}$. The damage variable $D_{l}$ is a non-dimensional value, it is equivalent to short crack $a_{l}$ discussed as reference (Yu $\& \mathrm{Bi}, 2009$ ). Here must be 
defined in $1 \mathrm{~m}$ (meter) equivalent to 1000 of damage-units, $1 \mathrm{~mm}$ (millimeter) equivalent to one of damage-unite, and must put up conversion of dimensions and units. It should point that units of the $d a_{1} / d N_{1}$ in Equation (1) and (6) are all $\mathrm{m} /$ cycle, and the units of the $d D_{1} / d N_{1}$ in (7) and (8) are unit number of damage per cycle $(D /$ cycle $)$.

\subsection{The Calculations of Rates at Second Stage under Mean Stress $\sigma_{m} \neq 0$ Condition}

In Figure 1, the beeline $C_{l} C_{2}$ via logarithm predigesting transacting can be represented as long crack growth behavior of material at the second stage. For its growth rate at this stage can be also adopted by above mentioned the multiplication parameters $\Delta \delta_{t} \cdot \Delta K_{1}$ to describe,

$$
\frac{d a_{2}}{d N_{2}}=A_{2}^{*} \times\left(\Delta Q_{2}\right)^{\frac{m_{2} \lambda_{2}}{m_{2}+\lambda_{2}}},(m / \text { clcle })
$$

Here

$$
\begin{aligned}
& Q_{2}=\delta_{t} \cdot K_{\mathrm{I}}\left(M P a \cdot m^{3 / 2}\right) \\
& \Delta Q_{2}=\Delta \delta_{t} \cdot \Delta K_{\mathrm{I}}\left(M P a \cdot m^{3 / 2}\right)
\end{aligned}
$$

For work stress $\sigma$ is less than yield stress $\sigma_{s}\left(\sigma<\sigma_{s}\right)(\mathrm{GB} / \mathrm{T} 19624-2004,2005)$.

$$
\begin{gathered}
\delta_{t}=\pi a_{2} \sigma_{s} \times\left(\sigma / \sigma_{s}\right)^{2} / E,(\%) \\
\Delta K_{\mathrm{I}}=\Delta K_{2}=F_{2} \Delta \sigma \sqrt{\pi a_{2}},(M P a \sqrt{m}) \\
\left.\Delta Q_{2}=\Delta \delta_{t} \cdot \Delta K_{\mathrm{I}}=\frac{\Delta \sigma^{3}}{4 \sigma_{s}^{2}} \times \sigma_{s} / E\right]\left(\sqrt{\pi a_{2}}\right)^{3},\left(M P a \cdot m^{3 / 2}\right)
\end{gathered}
$$

Here $F_{2}$ is the correction coefficient of concerning crack shape and size. $E$ is a modulus of elasticity. And for work stress is equal or greater than yield stress $\left(\sigma \geq \sigma_{s}\right)$

$$
\begin{gathered}
\delta_{t}=0.5 \pi \times \sigma_{s} \times a_{2}\left(\sigma / \sigma_{s}+1\right) / E, \\
\Delta Q_{2}=\left[0.5 \times \Delta \sigma \cdot \sigma_{s}\left(\sqrt{\pi a_{2}}\right)^{3}\left(\Delta \sigma / 2 \sigma_{s}+1\right)\right] / E
\end{gathered}
$$

Moreover,

$$
A_{2}^{*}=2\left[4\left(K_{2 c}-K_{2 m}\right)\left(\delta_{2 c}-\delta_{m}\right)\right]^{-\frac{m_{2} \lambda_{2}}{m_{2}+\lambda_{2}}} \times \frac{\left(a_{e f f}-a_{02}\right)}{\left(N_{p v}-N_{02}\right)},\left(m^{3 / 2} \cdot M P a\right)^{-\frac{m_{2} \lambda_{2}}{m_{2}+\lambda_{2}}} \times m / \text { cycle }
$$

Where the $Q_{2}$ and $\Delta Q_{2}$ are defined severally as the long crack stress-strain factor and stress-strain factor range, that are driving forces of long cracks growth at second stage. Their units are this $M P a \cdot m^{3 / 2} \cdot m_{2}$ and $\lambda_{2}$ are materials constants under high cycle or low cycle fatigue, respectively. $m_{2}=-1 / b_{2}{ }^{\prime}, b_{2}{ }^{\prime}$ is fatigue strength exponent under high cycle fatigue; $\lambda_{2}=-1 / c_{2}{ }^{\prime}, c_{2}$ ' is fatigue ductility exponent under low cycle fatigue. The parameter $A_{2}{ }^{*}$ is a comprehensive material constant at the second stage; it must be obtained from experiment. Its physical meaning is also a concept of power, is a maximal increment value gave out energy to cause fracture in one cycle; Its geometrical meaning is a maximal micro-trapezia area to approximate to beeline, also is an intercept between $\mathrm{O}_{3}-\mathrm{O}_{4}$ on the y-axis for beeline 2', it is also a projection of corresponding to curve $6\left(C_{1} k C_{2}\right)$ (Figure 1). Its slope of micro-trapezium bevel edge just is corresponding to the exponent $m_{2} \lambda_{2} /\left(m_{2}+\lambda_{2}\right)$ of formula (17). The comprehensive material constant $A_{2}{ }^{*}$ is also having function relation with other each parameter in (17). $K_{m}$ is a mean stress intensity factor. $\delta_{m}$ is a mean value of the crack tip open displacement, it can be also ignored, $\delta_{m} \approx 0$.

Where 


$$
v_{p v}=\frac{\left(a_{e f f}-a_{02}\right)}{\left(N_{p v}-N_{02}\right)} \approx 3 \times 10^{-8} \sim 3 \times 10^{-7}=v^{*},(\mathrm{~m} / \mathrm{cycle})
$$

Author research and think, the parameter $v_{p v}$ is a constant of rate at the second stage, it is defined to be the virtual rate, it is an equivalent rate caused in precrack, it can take same value by reference (Yaliema, 1981), $v_{p v}=v^{*}(m /$ cycle $) \cdot a_{e f f}$ is effective size during steady growth course for long crack, $a_{02}$ is precrack size. $N_{p v}$ is a virtual life, $N_{02}$ is initial life at the second stage, $N_{02}=0$. For the sake of safety, the comprehensive constant $A_{2}{ }^{*}$ in (17) should be calculated as following form,

$$
A_{2 e f f}^{*}=2\left[4\left(K_{2 e f f}-K_{2 m}\right)\left(\delta_{2 e f f}-\delta_{m}\right)\right]^{-\frac{m_{2} \lambda_{2}}{m_{2}+\lambda_{2}}} \times v_{p v},\left(m^{3 / 2} \cdot M P a\right)^{-\frac{m_{2} \lambda_{2}}{m_{2}+\lambda_{2}}} \times m / c y c l e
$$

Where $A_{2 e f f}^{*}$ is effective comprehensive material constant at the second stage, its physical meaning is maximal increment value given out effective power in one cycle, that is to pay an energy resisted external force. The $K_{2 e f f}$ is the effective value of stress intensity factor, $\delta_{2 e f f}$ is the effective value of the crack tip open displacement, that are the parameters during steady growth course for long crack, they must be obtained from experiment. Suppose that

$$
K_{2 e f f}=\sqrt{K_{1 c} \times K_{t h}} \text { or } \delta_{\text {eff }}=(0.15 \sim 0.55) \delta_{\mathrm{c}}
$$

*It must be substituted for $\frac{\left(a_{p v}-a_{01}\right)}{\left(N_{p v}-N_{01}\right)}$ by $\frac{\left(a_{e f f}-a_{02}\right)}{\left(N_{p v}-N_{02}\right)}$ in Equation (17) and (18).

So for $\sigma<\sigma_{s}$, its final expansion equation of the Equation (9) is as follow form,

$$
\frac{d a_{2}}{d N_{2}}=2\left[4\left(K_{2 c}-K_{2 m}\right)\left(\delta_{2 c}-\delta_{m}\right)\right]^{-\frac{m_{2} \lambda_{2}}{m_{2}+\lambda_{2}}} \times v_{p v}\left[\frac{(\Delta \sigma)^{3}}{4 E \sigma_{s}}\left(\sqrt{\pi a_{2}}\right)^{3}\right]^{\frac{m_{2} \lambda_{2}}{m_{2}+\lambda_{2}}},(m / \text { cycle })
$$

For $\sigma \geq \sigma_{s}$

$$
\frac{d a_{2}}{d N_{2}}=2\left[4\left(K_{2 c}-K_{2 m}\right)\left(\delta_{2 c}-\delta_{m}\right)\right]^{-\frac{m_{2} \lambda_{2}}{m_{2}+\lambda_{2}}} \times v_{p v} \times\left(\left[0.5 \times \Delta \sigma \cdot \sigma_{s}\left(\sqrt{\pi a_{2}}\right)^{3}\left(\Delta \sigma / 2 \sigma_{s}+1\right)\right] / E\right)^{m_{2} \lambda_{2}+\lambda_{2}},(m / \text { cycle })
$$

Accordingly, for $\sigma<\sigma_{s}$, the crack growth rate of long crack in effective region should become,

$$
\left(\frac{d a_{2}}{d N_{2}}\right)_{e f f}=2\left[4\left(K_{2 e f f}-K_{2 m}\right) \delta_{2 e f f}\right]^{-\frac{m_{2} \lambda_{2}}{m_{2}+\lambda_{2}}} \times v_{p v}\left[\frac{(\Delta \sigma)^{3}}{4 E \sigma_{s}}\left(\sqrt{\pi a_{2}}\right)^{3}\right]^{\frac{m_{2} \lambda_{2}}{m_{2}+\lambda_{2}}},(\mathrm{~m} / \mathrm{cycle})
$$

For $\sigma \geq \sigma_{s}$,

$$
\left(\frac{d a_{2}}{d N_{2}}\right)_{e f f}=2\left[4\left(K_{2 e f f}-K_{2 m}\right) \delta_{2 e f f}\right]^{-\frac{m_{2} \lambda_{2}}{m_{2}+\lambda_{2}}} \times v_{p v} \times\left[\left[0.5 \times \Delta \sigma \cdot \sigma_{s}\left(\sqrt{\pi a_{2}}\right)^{3}\left(\Delta \sigma / 2 \sigma_{s}+1\right)\right] / E\right]^{m_{2}+\lambda_{2}},(m / c y c l e)
$$

If use above mentioned same method, put up conversion for variable, then the damage evolving rate equation is deduced as following form

$$
\begin{aligned}
& \frac{d D_{2}}{d N_{2}}=A_{2}^{\prime *} \times\left(\Delta Q_{2}^{\prime}\right)^{\frac{m_{2} \lambda_{2}}{m_{2}+\lambda_{2}}},(D / \text { clcle }) \\
& \left(\frac{d D_{2}}{d N_{2}}\right)_{e f f}=A_{2 e f f}^{\prime *} \times\left(\Delta Q_{2}\right)^{\frac{m_{2} \lambda_{2}}{m_{2}+\lambda_{2}}},(\text { D/clcle })
\end{aligned}
$$

Corresponding equations with mentioned above are as follows, for $\sigma<\sigma_{s}$, 


$$
\Delta Q_{2}^{\prime}=\frac{(\Delta \sigma)^{3}}{4 E \sigma_{s}}\left(\sqrt{\pi D_{2}}\right)^{3},\left(M P a \cdot D^{3 / 2}\right)
$$

For $\sigma \geq \sigma_{s}$, it is

$$
\begin{gathered}
\Delta Q_{2}^{\prime}=\left[0.5 \times \Delta \sigma \cdot \sigma_{s}\left(\sqrt{\pi D_{2}}\right)^{3}\left(\Delta \sigma / 2 \sigma_{s}+1\right)\right] / E \\
A_{2}^{\prime *}=2\left[4\left(K_{2 c}^{\prime}-K_{2 m}^{\prime}\right)\left(\delta^{\prime}{ }_{2 c}-\delta^{\prime}{ }_{2 m}\right]^{-\frac{m_{2} \lambda_{2}}{m_{2}+\lambda_{2}}} \times v_{p v}\right. \\
A_{2 e f f}^{\prime^{*}}=2\left[4\left(K^{\prime}{ }_{2 e f f}-K^{\prime}{ }_{2 m}\right)\left(\delta^{\prime}{ }_{2 e f f}-\delta^{\prime}{ }_{2 m}\right)\right]^{-\frac{m_{2} \lambda_{2}}{m_{2}+\lambda_{2}}} \times v_{p v}
\end{gathered}
$$

Where $\Delta Q_{2}^{\prime}$ is defined to be "the damage stress-strain factor range" at crack growth stage, that is driving force to make material undergone to damage. The material constant $K_{2 c}^{\prime}=K^{\prime}{ }_{l c}$ is defined as the critical damage stress intensity factor. $K_{2 e f f}^{\prime}$ is the effective damage stress intensity factor. Their units are all this $M P a \sqrt{D}$. That $K^{\prime}{ }_{2 c}$ and $K^{\prime}{ }_{2 e f f}$ are respectively equivalent to $K_{2 c}, K_{\text {eff: }} \delta^{\prime}{ }_{2 c}$ and $\delta^{\prime}{ }_{2 e f f}$ are defined respectively as the critical damage crack tip open displacement and the effective damage crack tip open displacement, that are equivalent with $\delta_{c}$ and $\delta_{\text {eff. }}$. The units of other parameters in equations are also invariant. Therefore, if adopt critical $A_{2}{ }^{*}$, for $\sigma<\sigma_{s}$, their final expansion equations is as follow form,

$$
\frac{d D_{2}}{d N_{2}}=2\left[4\left(K_{2 c}^{\prime}-K_{2 m}^{\prime}\right) \delta_{2 c}^{\prime}\right]^{-\frac{m_{2} \lambda_{2}}{m_{2}+\lambda_{2}}} \times v_{p v} \times\left[\frac{(\Delta \sigma)^{3}}{4 E \sigma_{s}}\left(\sqrt{\pi D_{2}}\right)^{3}\right]^{\frac{m_{2} \lambda_{2}}{m_{2}+\lambda_{2}}}
$$

If adopt effective $A^{*}{ }_{2 e f f}$, it is

$$
\left(\frac{d D_{2}}{d N_{2}}\right)_{e f f}=2\left[4\left(K_{2 e f f}^{\prime}-K_{2 m}^{\prime}\right) \delta_{2 e f f}^{\prime}\right]^{-\frac{m_{2} \lambda_{2}}{m_{2}+\lambda_{2}}} \times v_{p v} \times\left[\frac{(\Delta \sigma)^{3}}{4 E \sigma_{s}}\left(\sqrt{\pi D_{2}}\right)^{3}\right]^{\frac{m_{2} \lambda_{2}}{m_{2}+\lambda_{2}}}
$$

If adopt critical $A_{2}{ }^{*}$ and for $\sigma \geq \sigma_{s}$, its final expansion equation is

$$
\frac{d D_{2}}{d N_{2}}=2\left[4\left(K_{2 c}^{\prime}-K_{2 m}^{\prime}\right)\left(\delta_{2 c}^{\prime}-\delta_{2 m}^{\prime}\right)\right]^{-\frac{m_{2} \lambda_{2}}{m_{2}+\lambda_{2}}} \times v_{p v} \times\left[0.5 \times \Delta \sigma \cdot \sigma_{s}\left(\sqrt{\pi D_{2}}\right)^{3}\left(\Delta \sigma / 2 \sigma_{s}+1\right) / E\right]^{\frac{m_{2} \lambda_{2}}{m_{2}+\lambda_{2}}}
$$

If not consider $\delta_{2 m}\left(\delta_{2 m}=0\right)$, adopt effective $A^{*}{ }_{2 e f f}$, that is

$$
\left(\frac{d D_{2}}{d N_{2}}\right)_{e f f}=2\left[4\left(K_{2 e f f}^{\prime}-K_{2 m}^{\prime}\right) \delta_{2 e f f}^{\prime}\right]^{-\frac{m_{2} \lambda_{2}}{m_{2}+\lambda_{2}}} \times v_{p v} \times\left[0.5 \times \Delta \sigma \cdot \sigma_{s}\left(\sqrt{\pi D_{2}}\right)^{3}\left(\Delta \sigma / 2 \sigma_{s}+1\right) / E\right]^{\frac{m_{2} \lambda_{2}}{m_{2}+\lambda_{2}}}
$$

It must be point that the units of crack growth rates are the m/cycle in Equation (9), (21) and (22); but the units of damage evolving rates are the unit number of damage per cycle (unit number of damage /cycle) in Equation (31-34).

\section{Calculations of Rates in Whole Process}

Should point, the calculations of rates in whole process can use two methods:

1) Use corresponding equation and its variable $a_{l}$ (or $\left.D_{l}\right)$ and $a_{2}$ (or $D_{2}$ ) by each stage respectively to calculate its rates, that is just above mentioned method.

2) Select varied corresponding equations in two stages, use same damage variable $\alpha$ or $D$ in whole process, by means of computing program complete the computing and the description of the whole curves. The positive curves 5 and $6\left(D B_{l} k^{\prime} C_{l} k C_{2}\right)\left(\sigma_{m} \neq 0\right)$ in positive direction coordinate system in Figure 1 just are describing the changing law of the rates in whole process. This is also as following mentioned method.

Thus, we are both able to adopt the variable $a_{1}, a_{2}$ or the variables $D_{1}, D_{2}$ to calculate the evolving rates for their curves by two stages severally; and can also adopt same $a$ or $D$ to describe its curves in overall process.

For $\sigma<\sigma_{s}$, if ignore influence of $\varepsilon_{m}$ and $\delta_{m}$, if adopt crack size $a$ as variable, then computing expression of crack 
growth rate in whole process is as following form,

$$
\begin{aligned}
& d a / d N=2\left[4\left(\sigma_{f}^{\prime}-\sigma_{m}\right) \varepsilon_{f}^{\prime}\right]^{-\frac{m_{1} m_{1}^{\prime}}{m_{1}+m_{1}^{\prime}}} \times v_{f} \times\left(\Delta \varepsilon_{p} \Delta \sigma\right)^{\frac{m_{1} m_{1}^{\prime}}{m_{1}+m_{1}^{\prime}}} \times a \\
& +2\left[4\left(K_{2 c}-K_{2 m}\right) \delta_{2 c}\right]^{-\frac{m_{2} \lambda_{2}}{m_{2}+\lambda_{2}}} \times v_{p v} \times\left[\frac{(\Delta \sigma)^{3}}{4 E \sigma_{s}}(\sqrt{\pi a})^{3}\right]^{\frac{m_{2} \lambda_{2}}{m_{2}+\lambda_{2}}}
\end{aligned}
$$

For $\sigma \geq \sigma_{s}$, it should be,

$$
\begin{aligned}
& d a / d N=2\left[4\left(\sigma_{f}^{\prime}-\sigma_{m}\right) \varepsilon_{f}^{\prime}\right]^{-\frac{m_{1} m_{1}^{\prime}}{m_{1}+m_{1}^{\prime}}} \times v_{f} \times\left(\Delta \varepsilon_{p} \Delta \sigma\right)^{\frac{m_{1} m_{1}^{\prime}}{m_{1}+m_{1}^{\prime}}} \times a \\
& +2\left[4\left(K_{2 c}-K_{2 m}\right) \delta_{2 c}\right]^{-\frac{m_{2} \lambda_{2}}{m_{2}+\lambda_{2}}} \times v_{p v} \times\left[0.5 \times \Delta \sigma \cdot \sigma_{s}(\sqrt{\pi a})^{3} \times\left(\Delta \sigma / 2 \sigma_{s}+1\right) / E\right]^{\frac{m_{2} \lambda_{2}}{m_{2}+\lambda_{2}}},(m / \text { cycle })
\end{aligned}
$$

If adopt the damage variable $D$, for $\sigma<\sigma_{s}$, it is as following form,

$$
\begin{aligned}
& d D / d N=2\left[4\left(\sigma_{f}^{\prime}-\sigma_{m}\right) \varepsilon_{f}^{\prime}\right]^{-\frac{m_{1} m_{1}^{\prime}}{m_{1}+m_{1}^{\prime}}} \times v_{f} \times\left(\Delta \varepsilon_{p} \Delta \sigma\right)^{\frac{m_{1} m_{1}^{\prime}}{m_{1}+m_{1}^{\prime}}} \times D \\
& +2\left[4\left(K_{2 c}-K_{2 m}\right) \delta_{2 c}\right]^{-\frac{m_{2} \lambda_{2}}{m_{2}+\lambda_{2}}} \times v_{p v} \times\left[\frac{(\Delta \sigma)^{3}}{4 E \sigma_{s}}(\sqrt{\pi D})^{3}\right]^{m_{2} \lambda_{2}}
\end{aligned}
$$

For $\sigma \geq \sigma_{s}$, it is

$$
\begin{aligned}
& d D / d N=2\left[4\left(\sigma_{f}^{\prime}-\sigma_{m}\right) \varepsilon_{f}^{\prime}\right]^{-\frac{m_{1} m_{1}^{\prime}}{m_{1}+m_{1}^{\prime}}} \times v_{f} \times\left(\Delta \varepsilon_{p} \Delta \sigma\right)^{\frac{m_{1} m_{1}^{\prime}}{m_{1}+m_{1}^{\prime}}} \times D \\
& +2\left[4\left(K_{2 c}-K_{2 m}\right) \delta_{2 c}\right]^{-\frac{m_{2} \lambda_{2}}{m_{2}+\lambda_{2}}} \times v_{p v} \times\left[0.5 \times \Delta \sigma \cdot \sigma_{s}(\sqrt{\pi D})^{3} \times\left(\Delta \sigma / 2 \sigma_{s}+1\right) / E\right]^{\frac{m_{2} \lambda_{2}}{m_{2}+\lambda_{2}}}
\end{aligned}
$$

\section{Calculation Example}

A pressure vessel is made with steel $16 \mathrm{MnR}$, its strength limit of material $\sigma_{b}=573 \mathrm{MPa}$, yield limit $\sigma_{s}=361 \mathrm{MPa}$, reduction of area is $\varphi=0.51$, modulus of elasticity $E=200000 \mathrm{MPa}$.

Calculation data at short-crack growth stage: cyclic strength coefficient $K^{\prime}=1165 \mathrm{MPa}$, strain-hardening exponent $n^{\prime}=0.187$; Fatigue strength coefficient $\sigma_{f}^{\prime}=947.1 \mathrm{MPa}$, fatigue strength exponent $b^{\prime}{ }_{1}=0.111, m_{1}=9.009$; Fatigue ductility coefficient $\varepsilon_{f}^{\prime}=0.464$, fatigue ductility exponent $c^{\prime}{ }_{1}=-0.5395, m^{\prime}{ }_{l}=1.8536$.

Calculation data at long-crack growth stage: Threshold level $\Delta K_{t h}=8.6 \mathrm{MPa} \sqrt{\mathrm{m}}$, critical stress intensity factor $K_{2 c}=K_{1 c}=927 M P a \sqrt{\mathrm{m}}$, critical damage stress intensity factor $K_{2 c}^{\prime}=92.7 \mathrm{MPa} \sqrt{D_{2}}$ of equivalent with the $K_{\mathrm{Ic}}\left(K_{2 c}\right)$.

Suppose shape correcting coefficient of long crack $F_{2}=1.12$. Its working stress in pressure vessel $\sigma_{\max }=280 M P, \sigma_{\min }=0$, local stress $840 M P a$ at stress concentration point (stress concentration coefficient $K_{\mathrm{t}}=3$ ). Other computing data is in Table 1.

Try to calculate respectively:

1) The growth rate $d a_{I} / d N_{l}$ at short crack $a_{01}=0.00036 m$ at first stage.

2) Damage evolving rate $d D_{I} / d N_{l}$ corresponding to $a_{01}=0.00036 \mathrm{~m}$ at first stage.

3) Te growth rate $d a_{2} / d N_{2}$ at long crack size $a_{2 e f f}=0.0026 \mathrm{~m}$ at the second stage.

4) Damage evolving rate $d D_{2} / d N_{2}$ corresponding to long crack size $a_{2 e f f}=0.0026 m$ at the second stage. 
Table 1. Calculation data

\begin{tabular}{cccccccc}
\hline$K_{1 c}, M P a \sqrt{m}$ & $K_{\text {eff }}, M P a \sqrt{m}$ & $K_{t h}, M P a \sqrt{m}$ & $v_{p v}$ & $m_{2}$ & $\delta_{c}, m$ & $\lambda_{2}$ & $F_{2}$ \\
\hline 92.7 & 28.23 & 8.6 & $2 \times 10^{-7}$ & 3.91 & 0.00018 & 2.9 & 1.12 \\
\hline
\end{tabular}

\subsection{Calculations for Relevant Parameters}

1) Calculations for stress-strain by reference (Lee \& Pan, 2011).

$$
\varepsilon=\frac{\sigma}{E}+\left(\frac{\sigma}{K^{\prime}}\right)^{\frac{1}{n^{\prime}}}=\frac{280}{200000}+\left(\frac{280}{1165}\right)^{\frac{1}{0.187}}=1.4^{-3}+4.886^{-4}=1.8886^{-3}
$$

Strain range: $\Delta \varepsilon=\varepsilon_{\max }-\varepsilon_{\min }=1.8886 \times 10^{-3}$

Calculation for mean stress

$$
\sigma_{m}=\frac{\sigma_{\max }+\sigma_{\min }}{2}=\frac{280+0}{2}=140,(M P a)
$$

Calculation of mean strain

$$
\varepsilon_{m}=\frac{\varepsilon_{\max }+0}{2}=\frac{1.8886 \times 10^{-3}+0}{2}=9.443 \times 10^{-4}
$$

2) Calculation for stress intensity factor range at second stage by (13)

$$
\begin{gathered}
K_{2}=K_{1}=F_{2} \times \sigma \times \sqrt{\pi a_{2}}=1.12 \times 280 \sqrt{3.1416 \times 0.001}=15.69,(\operatorname{MPa} \sqrt{m}) \\
\Delta K_{2}=K_{2 \max }-K_{2 \min }=15.69-0=15.69(\mathrm{MPa} \sqrt{m})
\end{gathered}
$$

Calculation for mean stress intensity factor

$$
K_{2 m}=\frac{K_{2 \max }+K_{2 \min }}{2}=\frac{15.69+0}{2}=7.845(\operatorname{MPa} \sqrt{m})
$$

3) Computing for crack tip open displacement range $\Delta \delta_{t}$ and mean $\delta_{t m}$ at second stage by (15)

$$
\Delta \delta_{t}=\frac{0.5 \pi \times \sigma_{s} \times a_{2}}{E}\left(\frac{\sigma_{\max }-\sigma_{\min }}{2 \times \sigma_{s}}+1\right)=\frac{0.5 \times 3.1416 \times 361 \times 0.001}{200000} \times\left(\frac{280-0}{2 \times 361}+1\right)=3.9349 \times 10^{-6}(\mathrm{~m})
$$

Calculation for mean crack tip open displacement at second stage

$$
\delta_{t m}=\frac{0.5 \pi \times \sigma_{s} \times a \times}{E}\left(\frac{\left(\sigma_{\max }+\sigma_{\min }\right) / 2}{\sigma_{s}}+1\right)=\frac{0.5 \times 3.1416 \times 361 \times 0.001}{200000} \times\left(\frac{(280+0) / 2}{361}+1\right)=3.935^{-6},(\mathrm{~m})
$$

4) Calculation for effective stress intensity factor by $K_{t h}$ - and $K_{l c^{-}}$data in Table 1

$$
K_{2 e f f}=\sqrt{K_{t h} K_{1 c}}=\sqrt{8.6 \times 92.7}=28.23(\operatorname{MPa} \sqrt{m})
$$

Take shape coefficient of long crack $F_{2}=1.12$

Calculation for effective crack size $a_{2 e f f}$ in effective life history at the second stage,

$$
a_{2 e f f}=\frac{\left(K_{e f f}\right)^{2}}{F^{2} \pi \Delta \sigma^{2}}=\frac{(28.23)^{2}}{1.12^{2} \times 3.1416 \times 280^{2}}=0.0026 \mathrm{~m}
$$




\subsection{Calculations for Short Crack Growth Rate at the First Stage}

Take stress concentration coefficient $K_{t}=3, \varphi=0.51$.

According to formulas (5), (4), calculate comprehensive material constant $A_{1}{ }^{*}$,

$$
\begin{gathered}
v_{f}=\ln [1 /(1-\varphi)]=\ln [1 /(1-0.51)]=0.7133,(\% / \text { cycle }) \\
A_{1}^{*}=2\left[4\left(\sigma_{f}^{\prime}-\sigma_{m}\right)\left(\varepsilon_{f}^{\prime}-\varepsilon_{m}\right)\right]^{-\frac{m_{1} m_{1}}{m_{1}+m_{1}}} \times v_{f}=2\left[4(947.1-140) \times\left(0.464-9.443 \times 10^{-4}\right)\right]^{-\frac{9.009 \times 1.8536}{9.009+1.8536}} \times 1 \times 10^{-3} \times 0.7133=1.92^{-8}
\end{gathered}
$$

By Equation (6), calculation for growth rate at short crack $a_{01}=0.00036 \mathrm{~m}$ at the first stage,

$$
\begin{gathered}
d a_{1} / d N_{1}=2\left[4\left(\sigma_{f}^{\prime}-\sigma_{m}\right)\left(\varepsilon_{f}^{\prime}-\varepsilon_{m}\right)\right]^{-\frac{m_{1} m_{1}}{m_{1}+m_{1}}} \times v_{f} \times K_{t}(\Delta \varepsilon \cdot \Delta \sigma)^{\frac{m_{1} m_{1}}{m_{1}+m_{1}}} \times a_{1}=1.92^{-8} \times 3\left(1.8886 \times 1^{-3} \times 280\right)^{\frac{9.0009+1.8536}{9.0336}} \times 0.00036 \\
=1.92^{-8} \times 3 \times 0.528808^{1.5374} \times 0.00036=2.335^{-11},(\mathrm{~m} / \text { cycle })
\end{gathered}
$$

By Equation (6), calculation for its growth rate at $a_{01}=0.001 \mathrm{~m}$ at the first stage

$$
\begin{gathered}
d a_{1} / d N_{1}=2\left[4\left(\sigma_{f}^{\prime}-\sigma_{m}\right)\left(\varepsilon_{f}^{\prime}-\varepsilon_{m}\right)\right]^{-\frac{m_{1} m_{1}}{m_{1}+m_{1}}} v_{f} \times K_{t}(\Delta \varepsilon \cdot \Delta \sigma)^{\frac{m_{m} m_{1}}{m_{1}+m_{1}}} \times a_{1}=1.92^{-8} \times 3\left(1.8886 \times 1^{-3} \times 280\right)^{\frac{9.009 \times 1.8536}{9.009+1.8536}} \times 0.001 \\
=1.92^{-8} \times 0.001 \times(0.528808)^{1.5374}=2.177^{-11}(\mathrm{~m} / \text { cycle })
\end{gathered}
$$

4.3 Calculations for Damage Rate at the First Stage

By Equation (8), its damage rate at $a_{01}=0.00036 \mathrm{~m}$ should be as follow

$$
\begin{gathered}
d D_{1} / d N_{1}=2\left[4\left(\sigma_{f}^{\prime}-\sigma_{m}\right)\left(\varepsilon_{f}^{\prime}-\varepsilon_{m}\right)\right]^{-\frac{m_{1} m_{1}^{\prime}}{m_{1}+m_{1}^{\prime}}} v_{f} \times K_{t}(\Delta \varepsilon \cdot \Delta \sigma)^{\frac{m_{1} m_{1}^{\prime}}{m_{1}+m_{1}^{\prime}}} \times D_{1} \\
=1.92 \times 1^{-8} \times 3\left(1.8886 \times 1^{-3} \times 280\right)^{\frac{9.009 \times 91.8536}{9.00956}} \times 0.00036 \\
=1.92 \times 1^{-8} \times 0.00036 \times(0.528808)^{1.5374} \\
\left.=2.335^{-11} \text { (thousand damage-unit } / \text { cycle }\right)
\end{gathered}
$$

By Equation (8), its damage rate at $a_{01}=0.001 \mathrm{~m}$ should be as follow,

$$
\begin{gathered}
d D_{1} / d N_{1}=2\left[4\left(\sigma_{f}^{\prime}-\sigma_{m}\right)\left(\varepsilon_{f}^{\prime}-\varepsilon_{m}\right)\right]^{-\frac{m_{1}^{\prime} m_{1}^{\prime}}{m_{1}+m_{1}}} v_{f} \times K_{t}(\Delta \varepsilon \cdot \Delta \sigma)^{\frac{m_{1}^{\prime} m_{1}^{\prime}}{m_{1}+m_{1}}} \times D_{1}=1.92 \times 1^{-8} \times 3\left(1.8886 \times 1^{-3} \times 280\right)^{\frac{9.009 \times 1.8536}{9.009+1.8536}} \times 0.001 \\
=1.92 \times 1^{-8} \times 0.001 \times(0.528808)^{1.5374}=2.177^{-11} \text { (thousand damage- unit / cycle) }
\end{gathered}
$$

4.4 Calculations for Long Crack Growth Rate at the Second Stage

Take stress concentration coefficient $K_{t}=3$ by table 1 , take $v_{p v}=2 \times 10^{-7}(\mathrm{~m} / \mathrm{cycle})$.

Calculate for comprehensive material constant by (17)

$$
\begin{gathered}
A_{2}^{*}=2\left[4\left(K_{2 c}-K_{2 m}\right)\left(\delta_{2 c}-\delta_{m}\right)\right]^{-\frac{m_{2} \lambda_{2}}{m_{2}+\lambda_{2}}} \times v_{p v}=2\left[4(92.7-7.845)\left(0.00018-3.935 \times 10^{-6}\right)\right]^{\frac{-3.91 \times 2.9}{3.91+2.9}} \times 2 \times 10^{-7} \\
\left.=4.358 \times 10^{-5},(\mathrm{MPa} \sqrt{\mathrm{m}} \cdot \mathrm{m})^{\frac{-m_{2} \lambda_{2}}{m_{2}+\lambda}} \cdot \mathrm{m} / \mathrm{cycle}\right)
\end{gathered}
$$

According to Equation (22), calculate for its growth rate at long crack $a_{02}=0.001 \mathrm{~m}$,

$$
\begin{aligned}
& \frac{d a_{2}}{d N_{2}}=A_{2}^{*} \times K_{t}\left[0.5 \times \Delta \sigma \cdot \sigma_{s} F_{2}\left(\sqrt{\pi a_{2}}\right)^{3}\left(\Delta \sigma / 2 \sigma_{s}+1\right) / E\right]^{\frac{m_{2} \lambda_{2}}{m_{2}+\lambda_{2}}} \\
& =4.358 \times 10^{-5} \times 3\left[0.5 \times 280 \times 361 \times 1.12(\sqrt{\pi 0.001})^{3} \times\left(\frac{280}{2 \times 361}+1\right) / 200000\right]^{\frac{3.91 \times 2.9}{3.91+2.9}} \\
& =4.358 \times 10^{-5} \times 3\left[6.9164^{-5}\right]^{1.665}=1.548 \times 10^{-11}(\mathrm{~m} / \mathrm{cycle})
\end{aligned}
$$

Calculate for its growth rate at $a_{02}=0.0026 m$ at the second stage 


$$
\begin{aligned}
& \frac{d a_{2}}{d N_{2}}=A_{2}^{*} \times K_{t}\left[0.5 \times \Delta \sigma \cdot \sigma_{s} \times F_{2}\left(\sqrt{\pi a_{2}}\right)^{3}\left(\Delta \sigma / 2 \sigma_{s}+1\right) / E\right]^{\frac{m_{2} \lambda_{2}}{m_{2}+\lambda_{2}}} \\
& =4.358 \times 10^{-5} \times 3\left[0.5 \times 280 \times 361 \times 1.12(\sqrt{\pi 0.0026})^{3} \times\left(\frac{280}{2 \times 361}+1\right) / 200000\right]^{\frac{3.91 \times 2.9}{3.91+2.9}} \\
& =4.358 \times 10^{-5} \times 3\left[2.9^{-4}\right]^{1.665}=1.6834 \times 10^{-10}(\mathrm{~m} / \mathrm{cycle})
\end{aligned}
$$

\subsection{Calculations for Damage Rate at the Second Stage}

According to Equation (33), its damage rate should be as follow,

$$
\begin{aligned}
& \frac{d D_{2}}{d N_{2}}=A_{2}^{*} \times K_{t}\left[0.5 \times \Delta \sigma \cdot \sigma_{s} F_{2}\left(\sqrt{\pi D_{2}}\right)^{3}\left(\Delta \sigma / 2 \sigma_{s}+1\right) / E\right]^{\frac{m_{2} \lambda_{2}}{m_{2}+\lambda_{2}}} \\
& =4.358 \times 10^{-5} \times 3\left[0.5 \times 280 \times 361 \times 1.12(\sqrt{\pi 0.001})^{3} \times\left(\frac{280}{2 \times 361}+1\right) / 200000\right]^{\frac{3.91 \times 2.9}{3.91+2.9}} \\
& \left.=4.358 \times 10^{-5} \times 3\left[6.9164^{-5}\right]^{1.665}=1.548 \times 10^{-11} \text { (thousand damage-unit / cycle }\right) \\
& \frac{d D_{2}}{d N_{2}}=A_{2}^{*} \times K_{t}\left[0.5 \times \Delta \sigma \cdot \sigma_{s} \times F_{2}\left(\sqrt{\pi D_{2}}\right)^{3}\left(\Delta \sigma / 2 \sigma_{s}+1\right) / E\right]^{\frac{m_{2} \lambda_{2}}{m_{2}+\lambda_{2}}} \\
& =4.358 \times 10^{-5} \times 3\left[0.5 \times 280 \times 361 \times 1.12(\sqrt{\pi 0.0026})^{3} \times\left(\frac{280}{2 \times 361}+1\right) / 200000\right]^{\frac{3.91 \times 2.9}{3.91+2.9}} \\
& =4.358 \times 10^{-5} \times 3\left[2.9^{-4}\right]^{1.665}=1.6834 \times 10^{-10}(\text { thousand damage-unit / cycle })
\end{aligned}
$$

Thus, here can be seen that transitional rate $(d a / d N)_{t r}$ for the transitional point near at crack size $a_{t r}=0.001 \mathrm{~m}$ are: one rate $d a_{l} / d N_{l}=2.177^{-11}(\mathrm{~m} / \mathrm{cycle})$, at the first stage; other rate $d a_{2} / d N_{2}=1.548 \cdot 10^{-11}(\mathrm{~m} / \mathrm{cycle})$, at second stage. This show between two data at same point is closer.

\section{Conclusions}

1). About relations between material properties and crack growth (damage) driving forces: Consider some characters of elastic-plastic materials, which they are having the dualities of both elastic properties and the plastic properties. Therefore, describe their mathematical models undergone to fatigue damages, also should be having such mathematical expressions to contain both stress component and strain component. So At first stage, for the short-crack stress-strain factor $Q_{l}$, that should be and can be defined as driving force of the short-crack growth; for the damage stress-strain factor $Q_{l}$, it should be and can be defined as driving force in damage evolving process at this stage. At second stage, for the long-crack stress-strain factor $Q_{2}$, that should also be and can also be defined as driving force of the long-crack growth; and for the damage stress-strain factor $Q_{2}$,' it should also be and can also be defined as driving force in damage evolving process at this stage.

2). About the relations between the mathematical models and damage evolving processes: Under identical loading, the differences between the mathematical models to describe the materials behaviors are due to make change of the stiffness of materials that are difference of degrees of undergone damages at varied stages. Therefore, the models of driving forces are become from the $Q_{I}\left(Q_{1}{ }^{\prime}\right)$ at crack forming stage to the $Q_{2}\left(Q_{2}{ }^{\prime}\right)$ at crack growth stage; the exponents in equations are become from $m_{1} \cdot m_{1}^{\prime} /\left(m_{1}+m_{1}{ }^{\prime}\right)$ to $m_{2} \cdot \lambda_{2} /\left(m_{2}+\lambda_{2}\right)$; the comprehensive material constants are become from $A_{1}{ }^{*}$ to $A_{2}{ }^{*}$; the slopes of the curves at each stages are also changed.

3). About physical and geometrical meanings of some key parameters: The parameter $A_{l}{ }^{*}$ at the first stage and $A_{2}{ }^{*}$ at the second stage, they are all comprehensive material constants, they are all to have functional relations with other parameters. Their physical meanings of $A_{1}{ }^{*}$ and $A_{2}{ }^{*}$ are a concept of power, just are a maximal increment value gave out energy to cause failure in one cycle; their geometrical meanings are a maximal micro-trapezia area to approximate to beeline, also are an intercept on the y-axis.

4). About conversion methods of variables, dimensions and units: make the crack growth rates to convert to the damage evolving rates, only for the crack size $a$ convert to the damage variable $D$, their dimensions and units of other parameters in equations are all to keep invariability, again via mathematical derivation and dimensional 
analysis, define their relative dimensions and units, and must make " $1 \mathrm{~mm}$ of crack size" equivalent to " 1 unit of damage value', "1m of crack size" equivalent to '1000 unit of damage value.

\section{Acknowledgements}

Author thanks sincerity the Zhejiang Province Natural Science fund Committee that gives to support and provide subsidization of research funds. Numbers of supporting project is Y1111267.

\section{References}

GB/T 19624-2004. (2005). Safety assessment for in-service pressure vessels containing defects. China, 24-26. (in Chinese).

Lee, Y. L., Pan, J., \& Hathaway, R. B. (2011). Fatigue Testing and Analysis Theory and Practice (pp. 163-175). By Zhang Ranzhi translating. Beijing, China: National Defense Industry Press. (In Chinese).

Morrow, J. D. (1968). Fatigue Design Handbook, Section 3.2, SAE Advances in Engineering (pp. 21-29). Warrenddale, PA: Society for Automotive Engineers.

Smith, K. N., Watson, P., \& Topper, T. H. (1970). A stress-strain function of the fatigue of metals. Journal of Material, 5(4), 767-778.

Yaliema, S. Ya. (1981). Correction about Paris's equation and cyclic intensity character of crack. J. Strength Problem, 147(9), 20-28. (In Russian).

Yu, Y. G. (2003). Fatigue Damage Calculated by the Ratio-Method to Materials and Its Machine Parts. Chinese Journal of Aeronautics, 16(3), 157-161. http://dx.doi.org/10.1016/S1000-9361(11)60177-5

Yu, Y. G. (2003). Fatigue damage of materials with Small Crack Calculated by the Ratio Method under Cycle Loading. Special Issue Containing the Proceeding of the Internation Symposium on Macro-, Meso-, Micro-, and Nano-Mechanics of Materials (MM2003, pp. 79-86.). Hong Kong: Trans Tech Publication Ltd.

Yu, Y. G., \& Liu, X. (2006). Studies and Applications of three Kinds of Computing Methods by Describing Damage Evolving Behaviors for Elastic-Plastic Materials. Chinese Journal of Aeronautics, 19(1), 52-58. http://dx.doi.org/10.1016/S1000-9361(11)60267-7

Yu, Y. G., \& Xu, F. (2007). Studies and Application of Computing Methods on Small Crack Growth Behaviors for Elastic-plastic Materials. Chinese Journal of Mechanical Engineering, 43(12), 240-245. (In Chinese). http://dx.doi.org/10.3901/JME.2007.12.240

Yu, Y. G., \& Zhao, E. J. (1999). Computing to Damage Evolving Rate under Symmetric Cyclic Loading. FATIGUE'99, Proceedings of the Seventh International Fatigue Congress, Beijing, China. 2, pp. 1137.

Yu, Y. G., Bi, B. X., Ma, Y. H., \& Xu, F. (2009). Damage Computing in Whole Evolving Process Actualized for the Materials Behaviors of Structure with Cracks to Use Software Technique. 12th International Conference on Fracture Proceeding. Author Index Y. Yangui. July 12-19, CD. Ottawa, Canada.

Yu, Y. G., Jiang, X. L., Chen, J. Y., \& Wu, Z. Y. (2002). The Fatigue Damage Calculated with Method of the Multiplication $\Delta \varepsilon_{e} \Delta \varepsilon_{p}$ Fatigue. Proceeding of the Eighth International Fatigue Congress, 5/5, 2815-2822. In: vStockholm, Sweden. EMAS.

Yu, Y. G., Li, Z. H., Bi, B. X., Ma, Y. H., \& Xu, F. (2007). To accomplish Integrity Computings of Structures and Materials with Computing program in Whole Evolving Process on Fatigue-Damage-Fracture. ENGINEERING STRUCTURAL INTEGRITY: RESEARCH, DEVELOPMENT AND APPLICATION, Proceedings of the UK Forum for Engineering Structural Integrity's Ninth International Conference on Engineering Structural Integrity Assessment. Beijing, 1, pp. 180-183.

Yu, Y. G., Liu, X., Zhang, C. S., \& Tan, Y. H. (2006). Fatigue damage calculated by Ratio-Method Metallic Material with small crack under un-symmetric Cyclic Loading. Chinese Journal of Mechanical Engineering, 19(2), 312-31. http://dx.doi.org/10.3901/CJME.2006.02.312

Yu, Y. G., Sun, Y. M., Ma, Y. H., \& Xu, F. (2011). The Computing of intersecting relations for its Strength Problem on Damage and Fracture to Materials with short and long crack. International Scholarly Research Network ISRN Mechanical Engineering. Retrieved from http://www.hindawi.com/isrn/me/ 


\section{Appendix}

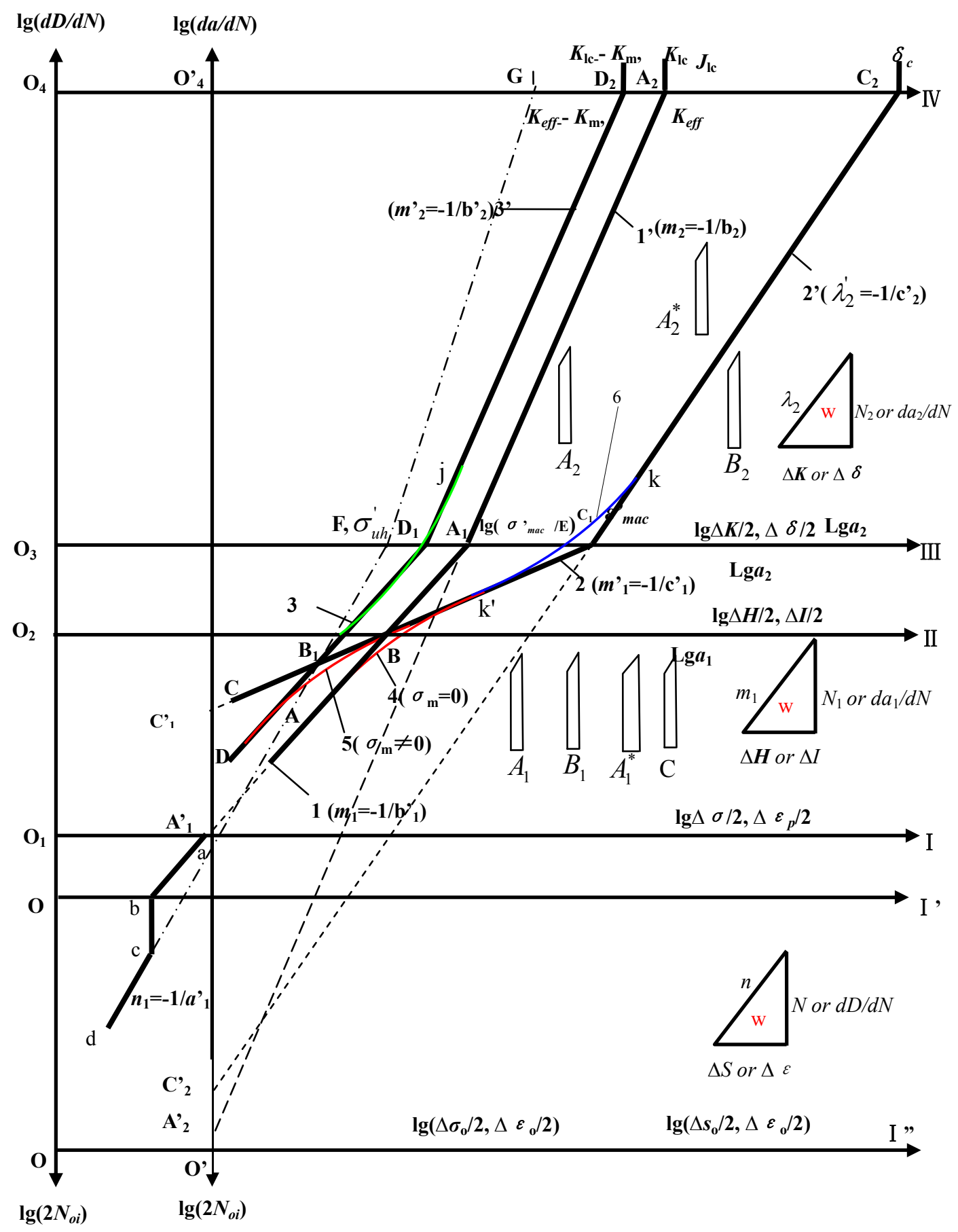

Figure 1. Bidirectional combined coordinate system and bidirectional simplified and schematic curves in the whole process (Combined cross figure of fatigue-damage-fracture) 